\title{
Computer simulation of high-energy-beam irradiation of $\beta$-cristobalite
}

\author{
Y. Sasajima ${ }^{1,2^{*}}$, H. Onuki $^{3}$, N. Ishikawa ${ }^{4}$, and A. Iwase ${ }^{5}$ \\ 1 Department of Materials Science and Engineering, Faculty of Engineering, Ibaraki University, 4-12-1 Nakanarusawa, Hitachi \\ 316-8511, Japan \\ 2 Frontier Research Center for Applied Atomic Sciences, Ibaraki University, Shirakata 162-4, Tokai 319-1106, Japan \\ 3 Graduate School of Science and Engineering, Ibaraki University, 4-12-1 Nakanarusawa, Hitachi 316-8511, Japan \\ 4 Japan Atomic Energy Agency (JAEA), Shirakata Shirane 2-4, Tokai 319-1195, Japan \\ 5 Department of Materials Science, Osaka Prefecture University, Gakuen-cho 1-1, Sakai, 599-8531, Japan \\ * Fax: 81(294)38-5226, e-mail: sasajima@mx.ibaraki.ac.jp
}

\begin{abstract}
The structural modification caused by the high-energy-beam irradiation of single-crystalline $\beta$-cristobalite was simulated by the molecular dynamics method. As the initial condition, high thermal energy was supplied to the individual atoms within a cylindrical region of nanometer-order radius located in the center of the specimen. The supplied thermal energy was first spent to change the crystal structure into an amorphous one within a short period of about $0.3 \mathrm{ps}$, then it dissipated in the specimen. The amorphous track radius $R_{a}$ was determined as a function of the effective stopping power $g S_{e}$, i.e., the thermal energy per unit length created by ion irradiation. It was found that the relationship between $R_{a}$ and $g S_{e}$ follows the relation $R_{a}^{2}=a \log \left(g S_{e}\right)+b$. Compared to the case of Si single crystal, it was easier to produce amorphous track because of the weakness of the bonding between $\mathrm{SiO}_{4}$ tetrahedra. It was also found that the mechanism of structural transition changes at $R_{a}=1 \mathrm{~nm}$. Key words: Computer simulation, high-energy-beam irradiation, $\beta$-cristobalite, molecular dynamics, thermal spike model
\end{abstract}

\section{INTRODUCTION}

For the development of materials with high resistance to radiation damage or with exotic properties produced by atomic-level modification, it is important to analyze the structural change when a solid is irradiated with a high-energy-beam . It is however very difficult to clarify the process because the change after the high-energy-beam irradiation is a nonequilibrium phenomenon with a very short relaxation time. Therefore, computer experiments based on the molecular dynamics (MD) method are regarded as a powerful tool to obtain complementary insights into real experiments. Bringa and coworkers have conducted such computer experiments in a series of studies [1-4]. Bringa and Johnson performed a MD simulation of the nonequilibrium energy transport from a cylindrical thermal spike in solid Ar [1]. They reported the following three points: (i) The thermal spike model cannot describe this energy transport. (ii) First, the supplied energy is transported to the nearest-neighbor region. (iii) Melt is created in the thermalized region immediately after the initial energy is supplied, then a pressure wave is transported to the nonmelting region. In one of their papers, they calculated the sputter yield of solid $\mathrm{Ar}$ and $\mathrm{O}_{2}$ after a cylindrical thermal spike was applied [2]. They found that the sputter yield is proportional to the effective stopping power $\mathrm{dE} / \mathrm{dx}$ (defined as "energy deposition per unit path length in solid" in their paper) in the high $\mathrm{dE} / \mathrm{dx}$ region. This result differs from both those of the thermal spike model and experiments. They pointed to the following reasons: (i) The thermal spike model incorrectly assumes thermal diffusion throughout the entire process. (ii) The process involving the supply of energy differs from that of real experiments. Bringa et al. derived sputtering yield from MD simulation assuming Lennard-Jones (LJ) and Morse potentials as the atomic interaction mechanism [3]. They examined the relationship between effective stopping power and sputtering yield and found that the yield scales with the binding energy and density of the material in the whole region of $\mathrm{dE} / \mathrm{dx}$. This means that the simulation results can be applied to a wide variety of materials. Recently, Bringa and Johnson reexamined the Coulomb explosion and thermal spike models on the basis of their computer simulation [4]. In the analysis of sputtering yield, the thermal spike model gives incorrect results because it assumes classical thermal diffusion throughout the entire process. At the first stage of ionized track formation, Coulomb explosion occurs, followed by the creation of a thermal spike. The yield is proportional to the square of ionization density $\mathrm{dJ} / \mathrm{dx}$ (note that $\mathrm{dJ} / \mathrm{dx}$ corresponds to the stopping power). This can explain experimental results.

There have been numerous studies based on MD simulation focused on sputtering [5-7]; however, the study of dynamic structural change in the specimen after high-energy-beam irradiation is limited to that on solid Ar conducted by Bringa and Johnson [1]. The topic is very important from the viewpoint of developing exotic materials using the track formation technique. We performed MD simulation of the structural relaxation caused by the high-energy-beam irradiation of single-crystalline $\mathrm{Si}[8]$. We found that the relationship 
between the amorphous track radius $R_{a}$ and the effective stopping power $g S_{e}$ follows the equation $R_{a}^{2}=a \log \left(g S_{e}\right)+b$ which is similar to the formula derived on the basis of the thermal spike model [9]. However, it is difficult to compare our results and actual experiment because of the difficulty of generating amorphous track in $\mathrm{Si}$. In the present study, the structural relaxation after the high-energy-beam irradiation of single crystalline $\beta$ cristobalite was simulated by the molecular dynamics method. The aims of this study are the following: (i) To clarify the details of the high-energy dissipation process following the structural change of the irradiated nanoscale region for a $\beta$-cristobalite single crystal.

(ii) To determine the correlation between the thermal energy given to the specimen and the radius of the track produced by the irradiation.

The obtained results were compared to those of Si single crystal obtained by our previous study [8].

\section{Method of simulation}

2.1 Molecular dynamics

The $\mathrm{Si}$ and $\mathrm{O}$ atoms were arranged to form the $\beta$ -cristobalite structure, in which $\mathrm{Si}$ atoms form a diamond lattice and $\mathrm{O}$ atoms sit at the midpoint of the $\mathrm{Si}-\mathrm{Si}$ nearest neighbor bonds. The bond length between $\mathrm{Si}$ and $\mathrm{O}$ was $0.174 \mathrm{~nm}$. The unit cell of $\beta$-cristobalite structure was repeated 7 times in $<100\rangle$ direction, 7 in $<010\rangle$ and 4 in $<001>$. The dimensions of the calculation region were $5.63 \mathrm{~nm} \times 5.63 \mathrm{~nm} \times 3.21$ $\mathrm{nm}$ and the number of atoms was 4704 in total. At first, the initial structure was relaxed by molecular dynamics method at $298 \mathrm{~K}$. Then, high thermal energy was given in the cylindrical region with radius $0.25-2.5 \mathrm{~nm}$ in the central part of the calculation region. The thermal energy is considered to be a part of energy deposited by irradiation of a heavy ion. This energy is called effective stopping power $g S_{e}$, which is thermal energy created from stopping power $S_{e}$. The factor $g$ represents the energy transfer ratio from stopping power to lattice vibration energy, i.e., thermal energy. Figure 1 shows the atomic structure viewed from $<001>$ just after the addition of high thermal energy.

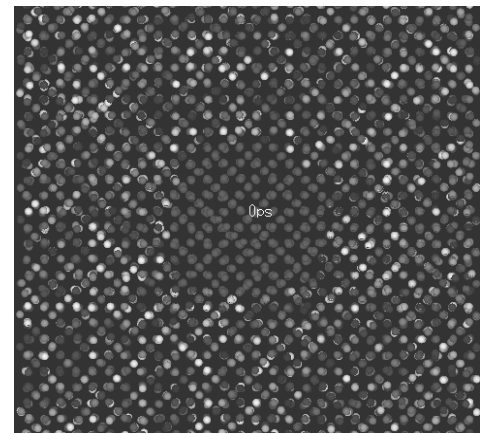

Fig.1 Atomic structure viewed from $<001>$ just after the addition of high thermal energy.

After that, the atomic motion was calculated by the molecular dynamics method, where the temperature of the peripheral region, the region outside the cylindrical region with radius $2.5 \mathrm{~nm}$, was kept at $298 \mathrm{~K}$ by velocity scaling method. The peripheral region can be considered as heat sink. Periodic boundary conditions were imposed in $\langle 100\rangle,<010\rangle$ and $<001\rangle$ directions. The velocity Verlet algorithm was used for the numerical integration of Newton's equation of motion [10]. The extended-Tersoff potential was used to calculate interactions between atoms [11, 12]. This potential is based on the Tersoff potential [13], and can reproduce the energy of a semiconductor, metal, oxide and other compounds. The functional form and parameters of the potential are given in references 11 and 12. The time step was $0.3 \mathrm{fs}$ and the duration of numerical experiment was 3 ps. The book-keeping and cell-index methods were used to reduce computing time [14].

\subsection{Method of structural analysis}

The calculated atomic structure was analyzed by visualization of the structure, calculation of radial distribution function and that of coordination number distribution. The radial distribution function and coordination number distribution were obtained for the two kinds of cylindrical region in the sample: central part of the sample and its peripheral region. Figure 2 shows the analyzed region viewed from $<001\rangle$ : the radius of the central part, hereafter called the center, is the same as the thermalized region explained in the previous section, and that of the peripheral region, hereafter called the layer, whose radius is two times that of the center.

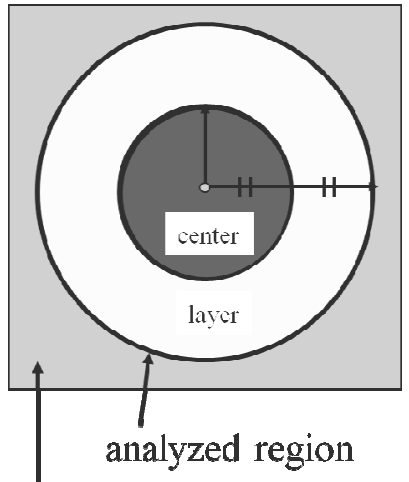

Heat sink (298K)

Fig.2 A schematic of the analyzed region, consisting of center and layer regions.

When the cylindrical amorphous region remained after the calculation, the track radius of its region was estimated by the $\mathrm{Si}$ atoms with coordination numbers other than 4 in the final structure. At first, the center of mass of the $\mathrm{Si}$ atoms with non-4-coordination number was calculated in two dimensional space, spanned by $<100>$ and $<010\rangle$. Then the number of the Si atoms with non-4-coordination number was calculated as a function of the two dimensional distance from the center of mass. The maximum value of the distance was considered to be track radius.

3. Results and discussion

3.1 Atomic structural analysis

Figures 3-1 to 3-3 show (a) the atomic structure, (b) the radial distribution function and (c) the distribution of coordination number, calculated from the beam 
irradiated sample with a thermalized region $1 \mathrm{~nm}$ in radius and with effective stopping power 0.06 $[\mathrm{keV} / \mathrm{nm}]$. For (b) and (c), coordination numbers were counted as the number of $\mathrm{Si}-\mathrm{O}$ bonds within the nearest neighbor $\mathrm{Si}-\mathrm{Si}$ bonds. In the case of single crystal of $\beta$-cristobalite, there are two peaks at 2 and 4 coordination number, corresponding to the number of $\mathrm{Si}$ around an $\mathrm{O}$ atom and that of $\mathrm{O}$ around a $\mathrm{Si}$ atom, respectively. The time durations are $0.03 \mathrm{ps}$ (just after the irradiation), $0.33 \mathrm{ps}$ and $3.00 \mathrm{ps}$ in this order. Figure 3-1 (a) and (b) are the same as the radial distribution function and the distribution of coordination

(a)

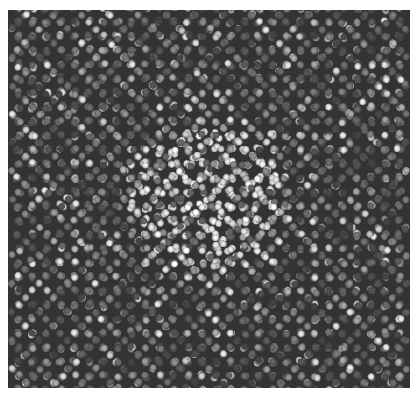

(b)

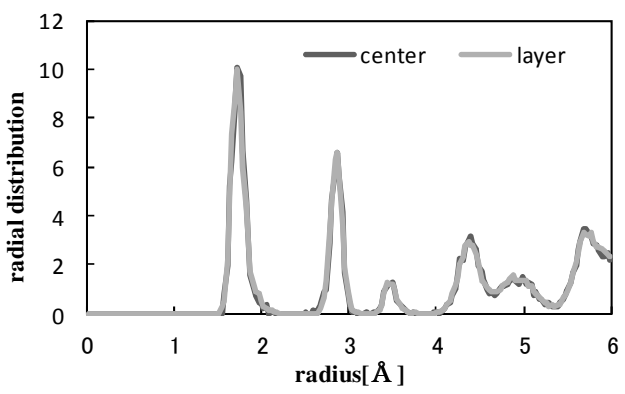

(c)

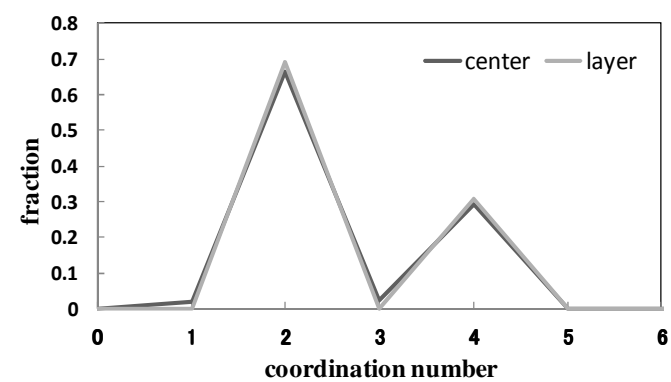

Fig.3-1 (a) The atomic structure, (b) the radial distribution function and (c) the distribution of coordination number, calculated from the beam irradiated sample with radius of the thermalized region 1 $\mathrm{nm}$ and with effective stopping power $0.06[\mathrm{keV} / \mathrm{nm}]$. The time duration is $0.03 \mathrm{ps}$, just after the structural relaxation at $297 \mathrm{~K}$. number calculated from the single crystal structure of $\beta$-cristobalite at $298 \mathrm{~K}$. These can be compared to the following results for the beam irradiation computer experiments shown in Figs. 3-2 to 3-3.

According to the high energy of the central region, the crystal structure was amorphized quickly as shown in Fig. 3-2 (b), at $0.3 \mathrm{ps}$ after the irradiation. Then, the structure partly recovers to reform crystalline regions by utilizing the residual thermal energy as shown in Fig. 3-3. However, the amorphous track remains in the central region of the sample as in Fig. 3-3.

(a)

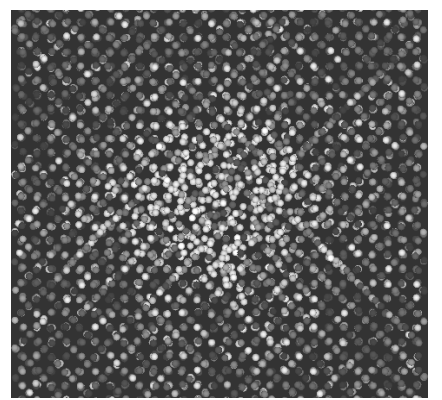

(b)

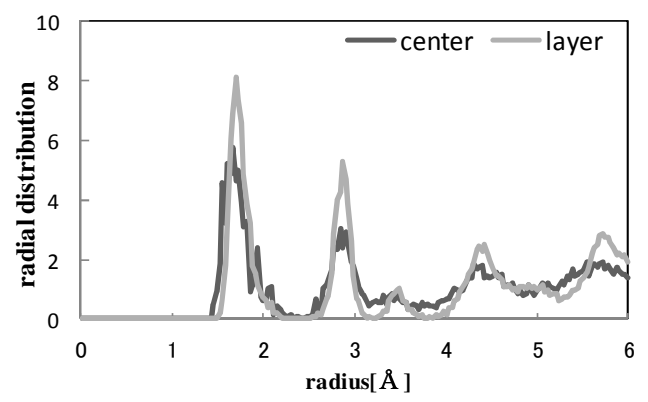

(c)

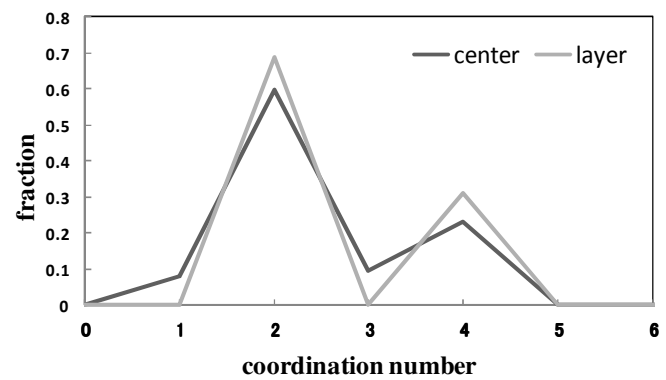

Fig.3-2 (a) The atomic structure, (b) the radial distribution function and (c) the distribution of coordination number, calculated from the beam irradiated sample with radius of the thermalized region 1 $\mathrm{nm}$ and with effective stopping power $0.06[\mathrm{keV} / \mathrm{nm}]$. The time duration is $0.33 \mathrm{ps}$. 
The prominent feature of the amorphous structure can be seen both in the radial distribution function and distribution of coordination numbers. In the distribution function, diffuseness of the fourth peak and distinctiveness of the fifth peak are seen in the case of amorphous regions. In the distribution of coordination numbers, the coordination numbers other than 2 and 4 , i.e., 1 and 3 can be seen in the amorphous structure.

(a)

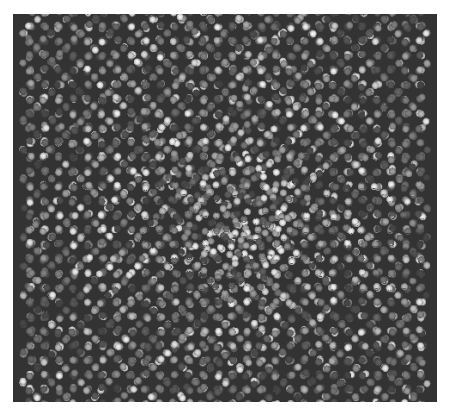

(b)

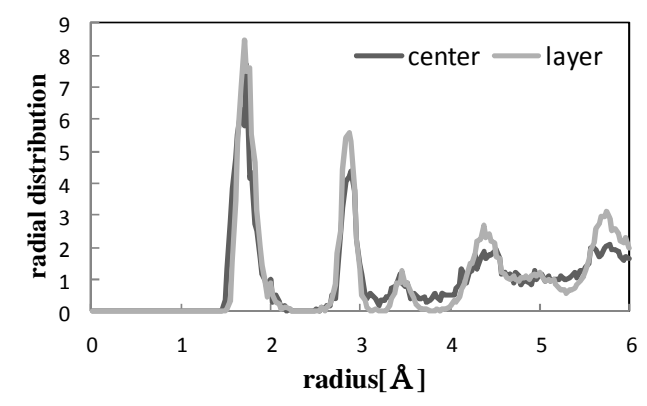

(c)

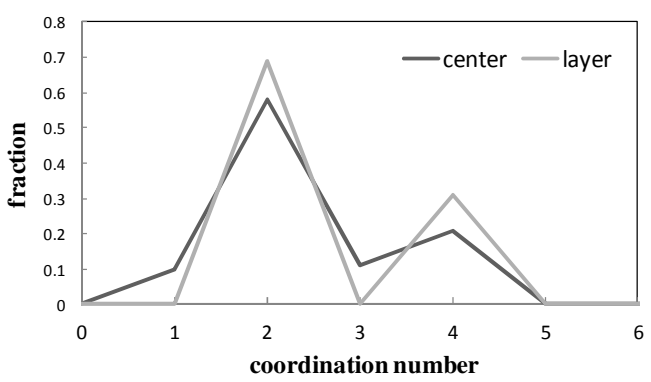

Fig.3-3 (a) The atomic structure, (b) the radial distribution function and (c) the distribution of coordination number, calculated from the beam irradiated sample with radius of the thermalized region 1 $\mathrm{nm}$ and with effective stopping power $0.06[\mathrm{keV} / \mathrm{nm}]$. The time duration is $3.00 \mathrm{ps}$
3.2 Threshold energy to create amorphous track

Varying the radius of thermalized region $\left(R_{t h}\right)$ and effective stopping power $\left(g S_{e}\right)$, the creation of amorphous track was judged by structural analysis described in the previous section. The obtained results are summarized in Fig. 4 (a). The results for Si single crystal are shown in Fig.4 (b) for comparison.

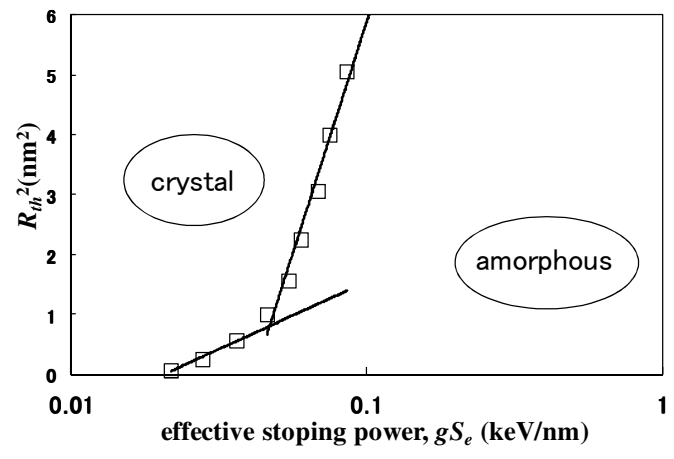

Fig.4(a) Structural map of irradiated system for $\beta$-cristobalite.

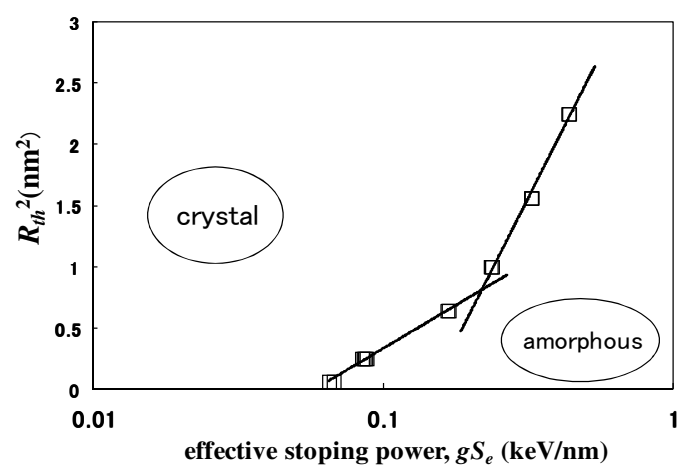

Fig.4(b) Structural map of irradiated system for Si.

In these figures, "crystal" means that the final structure is recystallized structures and "amorphous" that the cylindrical amorphous region remains in the sample. It can be seen that the threshold energy to create amorphous track for $\beta$-cristobalite is about one-fifth of that for $\mathrm{Si}$ single crystal. For example, at $R_{t h}=1 \mathrm{~nm}, \quad$ the threshold energy $\left(E_{t h}\right)$ for $\beta$-cristobalite is $0.0445 \mathrm{keV} / \mathrm{nm}$ and that for $\mathrm{Si}$ is $0.236 \mathrm{keV} / \mathrm{nm}$. The melting point $\left(T_{m}\right)$ of $\beta$-cristobalite is $1883 \mathrm{~K}$ and that of $\mathrm{Si}$ is $1683 \mathrm{~K}$. If we define the dimensionless parameter $\varepsilon$ as

$$
\varepsilon=\frac{E_{t h}}{k_{B} T_{m}}
$$

where $k_{B}$ is the Boltzmann constant, this parameter estimates the ease of amorphous track creation without the effect of difference of melting temperature. $\varepsilon$ for $\beta$-cristobalite is 267 and that for $\mathrm{Si}$ is 1573 for $R_{t h}=1$ $\mathrm{nm}$. Therefore amorphous track is created more easily in $\beta$-cristobalite than in $\mathrm{Si}$. As the energy of $\mathrm{Si}-\mathrm{O}$ 
bond in $\beta$-cristobalite is much stronger than that of $\mathrm{Si}$ Si bond in Si single crystal, this result is curious at first glance. However, it can be considered that the stable $\mathrm{SiO}_{4}$ tetrahedra remain and orientational order of the tetrahedra is lost in the amorphous region in $\beta$-cristobalite. From Fig. 3-3 (b) and (c), the first peak of radial distribution function and fraction of 4 coordinated atoms ( which are from the nearest bonding between $\mathrm{Si}$ and $\mathrm{O}$ ) are almost same as that of bulk. These facts indicate that $\mathrm{SiO}_{4}$ tetrahedra remain in the amorphous region.

3.3 Relationship between track radius and effective stopping power

Varying the radius of thermalized region $\left(R_{t h}\right)$ and effective stopping power $\left(g S_{e}\right)$, the radius of amorphous track $\left(R_{a}\right)$ was estimated by structural analysis described in section 3-1. The obtained results are summarized in figure 5 (a). The trend of $R_{a}-g S_{e}$ curve changes around $R_{a}=1 \mathrm{~nm}$ and effect of the difference of $R_{t h}$ is weak. Figure 5 (b) shows the fitted lines for the two types of the trend, $R_{a}<1 \mathrm{~nm}$ and $R_{a}>1 \mathrm{~nm}$. The result for $R_{t h}=1 \mathrm{~nm}$ is also shown, which covers the two types of fitted lines.

(a)

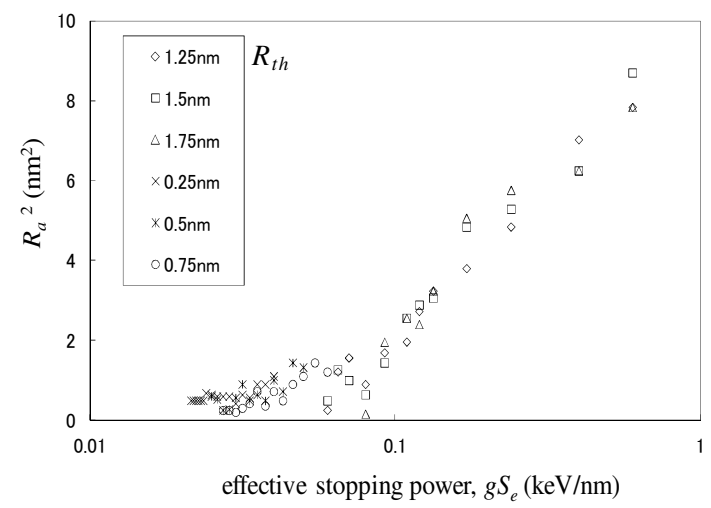

(b)

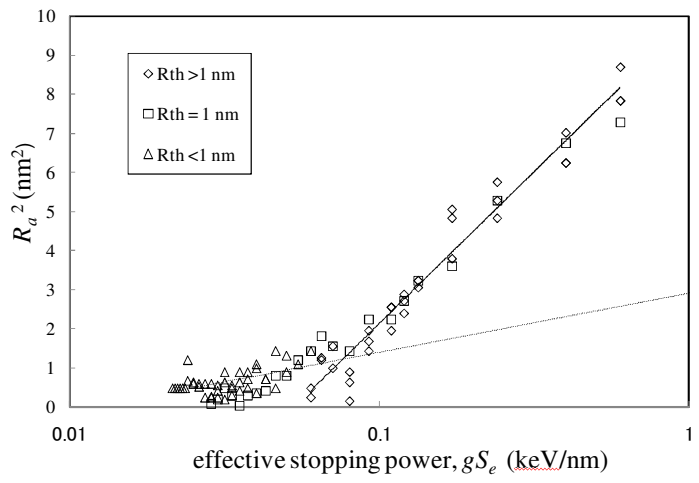

Fig.5 Relationship between track radius and effective stopping power. Data plotted for (a) $R_{t h}=0.25-1.75$ $\mathrm{nm}$ and (b) $R_{t h}>1 \mathrm{~nm}, R_{t h}=1 \mathrm{~nm}, R_{t h}<1 \mathrm{~nm}$ with fitted lines for $R_{a}<1 \mathrm{~nm}$ and $R_{a}>1 \mathrm{~nm}$.
The fitted functions are as follows,

$$
\begin{array}{ll}
R_{a}^{2}=0.657 \ln \left(g S_{e}\right)+2.91 & R_{a}<1 \mathrm{~nm} \\
R_{a}^{2}=3.37 \ln \left(g S_{e}\right)+9.90 & R_{a}>1 \mathrm{~nm}
\end{array}
$$

The result for $\beta$-cristobalite is compared to that for $\mathrm{Si}$ with $R_{t h}=1 \mathrm{~nm}$ as shown in Fig. 6 .

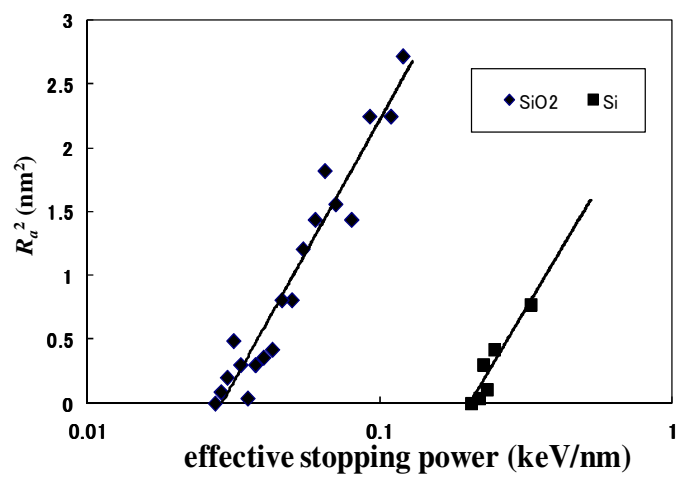

Fig.6 Relationship between track radius and effective stopping power for $\beta$-cristobalite and for $\mathrm{Si}$ with $R_{t h}=1$ nm.

These systems obey the same function such that

$$
R_{a}^{2}=a \ln \left(g S_{e}\right)+b
$$

where constants $a$ and $b$ were determined as $a=$ 1.79 and $b=6.32$ for $\beta$-cristobalite and $a=1.67$ and $b=2.66$ for Si. From this result, it can be seen that the effective stopping power necessary to generate amorphous tracks in $\beta$-cristobalite is much smaller than in Si.

We examined the system size and calculation time dependences on the radius of the created amorphous track in the $\mathrm{Si}$ case for $R_{t h}=1.5 \mathrm{~nm}$ and $g S_{e}=0.540 \mathrm{keV} / \mathrm{nm}$ (see Fig. 4 in reference 8 ). The amorphized track radius $R_{a}$ decreased by only $5 \%(1.27-1.21 \mathrm{~nm})$ after enlarging the system size and extending the duration of calculation by a factor of 1.5. This is the most severe case for the calculation; thus, the data obtained here are considered to be reliable.

The relationship between track radius $R_{a}$ and stopping power $S_{e}$ was obtained by actual experiments [15]. The results are shown in Fig. 7 with the simulation data for $R_{t h}=1 \mathrm{~nm}$. It should be noted that track radius is plotted as a function of effective stopping power $g S_{e}$, i.e. $S_{e}$ multiplied by the factor $g$. From this figure, we can estimate the value of $g$ of about 0.1 .

However, the direct comparison is difficult because of the difference of system dimension and $R_{t h}$. It is our next objective to simulate larger systems for direct comparison with actual experiments. 


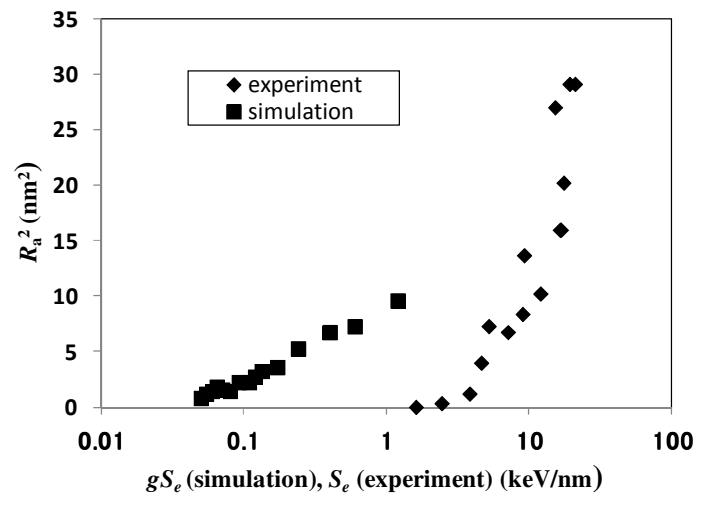

Fig.7 Relationship between track radius and effective stopping power(simulation) / stopping power (actual experiment) for $R_{t h}=1 \mathrm{~nm}$.

\section{Conclusion}

The structural relaxation caused by the high-energy-beam irradiation of single-crystalline $\beta$-cristobalite was simulated by the molecular dynamics method. According to the high energy of the central region, the crystal structure was amorphized quickly at 3 ps after the irradiation. Then, the structure partly recovers to crystal structure by utilizing the residual thermal energy in the system. However, the amorphous track remains in the central region. Varying the radius of thermalized region $\left(R_{t h}\right)$ and effective stopping power, the creation of amorphous track was analyzed. It can be seen that the threshold energy to create amorphous track for $\beta$-cristobalite is about one-fifth of that for $\mathrm{Si}$ single crystal. For example, at $R_{t h}=1 \mathrm{~nm}$, the threshold energy $\left(E_{t h}\right)$ for $\beta$-cristobalite is 0.0445 $\mathrm{keV} / \mathrm{nm}$ and that for $\mathrm{Si}$ is $0.236 \mathrm{keV} / \mathrm{nm}$. It can be considered that the stable $\mathrm{SiO}_{4}$ tetrahedra remains and orientational order of the tetrahedra is lost in the amorphous region in $\beta$-cristobalite.

Varying the radius of thermalized region $\left(R_{t h}\right)$ and effective stopping power $\left(g S_{e}\right)$, the radius of amorphous track $\left(R_{a}\right)$ was estimated. The trends of $R_{a}-g S_{e}$ curve change around $R_{a}=1 \mathrm{~nm}$ and effect of the difference of $R_{t h}$ is weak. The fitted functions are :

$$
\begin{array}{ll}
R_{a}^{2}=0.657 \mathrm{n}\left(g S_{e}\right)+2.91 & R_{a}<1 \mathrm{~nm} \\
R_{a}^{2}=3.37 \ln \left(g S_{e}\right)+9.90 & R_{a}>1 \mathrm{~nm}
\end{array}
$$

From the comparison of actual experiment, the factor $g$ can be estimated to be about 0.1 .

\section{Acknowledgements}

This research is financially supported by Grant-in-Aid for Scientific research under Contract No. 21360469. Dr. Tomoaki Akabane (a former graduate student of Ibaraki University, currently at Research Institute of System Planning, Inc. /ISP) is also acknowledged for the development of the program of molecular dynamics simulation. Mr. Takuya Osada and Mr. Naoki Ajima performed a part of the simulation.

\section{References}

[1] E. M. Bringa, and R. E. Johnson, Nucl. Instrum. Methods Phys. Res. B, 143, 513 - 535 (1998).

[2] E. M. Bringa, and R. E. Johnson: Nucl. Instrum. Methods Phys. Res. B, 152, 267 - 290 (1999).

[3] E. M.Bringa, R. E. Johnson, and M. Jakas, Phys. Rev. $B, \mathbf{6 0}, 15107$ - 15116 (1999).

[4] E. M. Bringa, and R. E. Johnson, Phys. Rev. Lett. , 88, 165501-1 - 165501-4 (2002).

[5] H. M. Urbassek, Nucl. Instrum. Methods Phys. Res. $B$, 122, 427-441 (1997).

[6]M. H. Shapiro, Radiation Effects and Defects in Solids, 142, 259-285 (1997).

[7]R. Smith, S. D. Kenny, and D. Ramasawmy, Phil. Trans. R. Soc. Lond. A, 362, 157-176 (2004).

[8] Y. Sasajima, T. Akabane, T. Nakazawa, and A. Iwase, Nucl. Instrum. Methods Phys. Res. B, 264, 259-266 (2007).

[9] G. Szenes, J. Nucl. Mater., 336, 81-89 (2005).

[10] L. Verlet, Phys. Rev. , 159, 98 - 103 (1967).

[11] A. Yasukawa, JSME Int. J. Ser. A, 39, 313-320 (1996).

[12] T. Iwasaki, and H. Miura, J. Mater. Res., 16, 1789-1794 (2001).

[13] J. Tersoff, Phys. Rev. B, 37, 6991 - 7000 (1988).

[14] D. C. Rapaport, "The art of molecular dynamics simulation", 2nd ed., Cambridge University Press, (2004).

[15] A. Meftah, F. Brisard, J. M. Costantini, E. Dooryhee, M. Hage-Ali, M. Hervieu, J. P. Stoquert, F. Studer, and M. Toulemonde, Phys. Rev. B, 49, $12457-$ 12463 (1994).

(Received March 21, 2013; Accepted July 26, 2013) 